Published online 2017 April 13.

Abstract

\title{
How Low Can We Go Dose Saving Through Low-Dose Examination for Urolithiasis Via Modern Computer Tomography with Tinfilter
}

\author{
Youssef Erfanian, ${ }^{1,}$ Nika Guberina, ${ }^{1}$ Saravanabavaan Suntharalingam, ${ }^{1}$ Jens Theysohn, ${ }^{1}$ and Johannes \\ Haubold \\ ${ }^{1}$ University Hospital Essen/ Germany \\ Corresponding author: Youssef Erfanian. E-mail: Youssef.Erfanian@uk-essen.de
}

Received 2016 December 21; Accepted 2017 February 08.

\begin{abstract}
Objectives: To compare radiation dose and image quality of three different CT devices using low dose abdominal protocols in patients with suspected urolithiasis. Differences in radiation dose due to usage of additional hardware (tin filter) and software (newer generation iterative reconstruction) were evaluated.

Methods: Low - dose examinations from two standard CT (siemens AS +, siemens flash) devices with a tin - filter CT (siemens force) were compared regarding dose-length product (DLP) and computed tomography dose index volume (CTDIvol). Image quality of each CT scan was assessed using a five point Likert scale $(0=$ major blurring, $4=$ excellent depiction). Kruskal - Wallis analysis was performed to test for significant subgroup differences in DLP, CTDIvol and image quality. An interrater agreement concerning image quality was evaluated.

Results: CT examinations of a total of 143 patients were assessed. DLP of Force was $56 \%$ and $55 \%$ lower in comparison to AS+ and flash, (74.3, 166.1 and 164.6 m Gycm respectively; $\mathrm{P}<0.001)$. CTDIvol of Force showed $60 \%$ and $58 \%$ significantly lower values as compared to AS + and Flash, respectively (1.4 vs. 3.5vs. $3.3 \mathrm{mGy} ; \mathrm{P}<0.001$ ). Image quality in force proved to be slightly higher as compared to flash and AS + (3.67, 3.50 and 3.59 respectively; P > 0.05). Interrater agreement regarding image quality was substantial for all CT devices $(\kappa=0.75, \mathrm{n}=143)$.

Conclusions: Modern CTs with the innovative technology of a built-in tin filter allow for significant reduction of radiation exposure in patients with suspected urolithiasis by optimizing the X - Ray spectrum with image quality as high as in standard CT devices
\end{abstract}

This is an abstract presented in the 33rd Iranian congress of radiology (ICR) and the 15th congress of Iranian radiographic science association (IRSA). 